\title{
Acidosis láctica severa asociada a infusión de propofol. Caso clínico
}

\author{
Carlos Romero $\mathbf{P}$, Mónica Morales $\mathbf{R}^{\mathrm{a}}$, Luisa Donaire $\mathbf{R}^{\mathbf{b}}$, \\ O svaldo Llanos V, Rodrigo Cornejo R, Ricardo Gálvez A, \\ José Castro 0.
}

\section{Severe lactic acidosis caused by propofol infusion. Report of one case}

\begin{abstract}
Propofol infusion syndrome (PRIS) is a rare but potentially lethal complications. This disorder is triggered under unknown circumstances by a propofol infusion of more than $5 \mathrm{mg} / \mathrm{kg} / \mathrm{h}$ for more than $48 \mathrm{~h}$. PRIS is characterized by a multiorgan failure and rhabdomyolysis and is induced by a disturbance in mitochondrial long chain fatty acid oxidation. We report a 43 year-old woman who underwent brain surgery due to a vascular malformation. In the immediate postoperative period, she had an unexplained and severe lactic acidosis. During anaesthesia, she received a propofol infusion of $7 \mathrm{mg} / \mathrm{kg} / \mathrm{h}$ that continued in the UCI at a rate of $3.5 \mathrm{mg} / \mathrm{kg} / \mathrm{h}$, for 8 hours more. The suspicion of PRIS motivated immediate discontinuation of propofol with rapid correction of lactic acidosis and full recovery of the patient (Rev Méd Chile 2008; 136: 88-92).
\end{abstract}

(Key words: Acidosis, lactic; Propofol; Rhabdomyolysis)
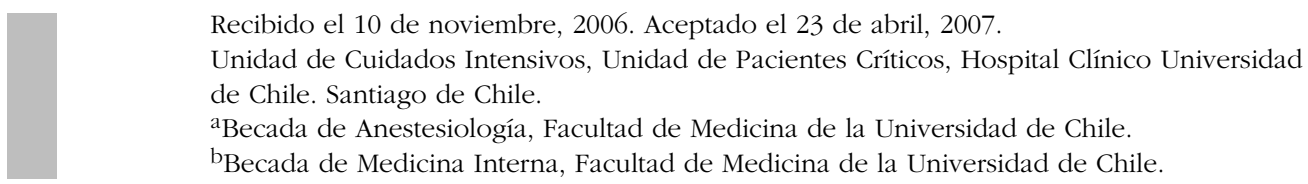

$\mathrm{E}$ propofol es un alquilfenol altamente liposoluble, usado frecuentemente en procedimientos quirúrgicos y para sedación de pacientes críticos. Las dosis habitualmente empleadas en pabellón oscilan entre 5 y $44 \mathrm{mg} / \mathrm{kg} / \mathrm{h}^{1}$. El propofol ha sido usado en anestesia durante más de 20 años y ha demostrado tener un buen perfil de seguridad ${ }^{2}$. Sin embargo, varios reportes han advertido sobre los peligros de su administración en altas dosis y por tiempo prolongado, tanto en niños $^{3-8}$ como en adultos ${ }^{9-13}$.

Correspondencia a: Dr. Carlos Romero P.

E mail: caromero@redclinicauchile.cl
El síndrome por infusión de propofol (SIP) es una entidad de presentación infrecuente caracterizada por el desarrollo de acidosis láctica severa, rabdomiolisis, disfunción cardiaca, hepática y renal e incremento de la lipemia. En su estadio avanzado, puede derivar en bradicardia aguda, que suele ser refractaria al tratamiento, y que puede progresar a asistolia ${ }^{14,15}$. No todos los enfermos expresan la forma completa del síndrome; de ahí la importancia de tenerlo siempre presente dentro de los diagnósticos diferenciales, ya que una oportuna intervención puede revertir las alteraciones metabólicas e impedir un desenlace fatal.

Presentamos el caso de una paciente joven sometida a una intervención neuroquirúrgica elec- 
tiva, quien recibió una infusión de propofol en dosis altas, pero sólo por un corto período de tiempo, y que desarrolló una acidosis láctica severa asociada a la infusión de este agente.

\section{Caso Clínico}

Se trata de una mujer de 43 años de edad con antecedentes de cefalea y crisis convulsivas generalizadas tónico clónicas de reciente inicio. Debido a su sintomatología es sometida a estudio y se diagnóstica la presencia de una malformación arteriovenosa (MAV) perirrolándica (Spetzler III), dependiente de la arteria cerebral media derecha. Dadas las características de la lesión se decidió realizar tratamiento quirúrgico. La paciente recibía tratamiento anticonvulsivante con ácido valproico debido a que era alérgica a la fenitoína.

El 13 de junio de 2006 a las 08:00 horas, fue sometida a una craneotomía parietal derecha más resección de la MAV. Durante las 7 horas que duró el procedimiento quirúrgico, se administraron 7 $\mathrm{mg} / \mathrm{kg} / \mathrm{h}(117 \mathrm{mg} / \mathrm{kg} / \mathrm{min})$ de propofol y $0,25 \mathrm{mg} /$ $\mathrm{kg} / \mathrm{min}$ de remifentanil. La exéresis de la MAV pudo ser ejecutada en forma satisfactoria y sin incidentes intra-operatorios. En el período postoperatorio inmediato (estando aún en pabellón), la paciente presentó una crisis convulsiva tónico clónica generalizada que cedió con la administración de $4 \mathrm{mg}$ de lorazepam por vía endovenosa.

A las 16:00 horas del mismo día y una vez estabilizada, la paciente es trasladada desde pabellón a la Unidad de Cuidados Intensivos (UCI), donde se mantiene en ventilación mecánica y se continúan las medidas de neuroprotección habituales, incluida la sedo-analgesia (midazolam/ fentanil) y el control de las crisis convulsivas con ácido valproico.

Los exámenes de química sanguínea se encontraban dentro de rangos normales, sin disfunción renal, hepática o de coagulación y sin evidencia de infección activa (Tabla 1). Sin embargo, a las 17:00 destacaba la presencia de una acidosis láctica incipiente con $\mathrm{pH} 7,34 ; \mathrm{PaCO}_{2} 36 \mathrm{mmHg}$, Bicarbonato $19 \mathrm{mmol} / \mathrm{L}$; $\mathrm{BE}-6,4 \mathrm{mmol} / \mathrm{L}$ y ácido

Tabla 1. Q uímica sanguínea pre y posquirúrgica

\begin{tabular}{|c|c|c|c|c|}
\hline Variables & $\begin{array}{l}\text { Pre cirugía } \\
06 / 03 / 2006\end{array}$ & $\begin{array}{l}\text { Ingreso UCI } \\
13 / 06 / 2006\end{array}$ & $\begin{array}{l}12 \text { horas } \\
\text { Postingreso } \\
14 / 06 / 2006\end{array}$ & $\begin{array}{l}\text { Rangos normales } \\
\text { (Unidad de medida) }\end{array}$ \\
\hline Creatinina & 0,7 & 0,6 & 0,6 & $0,7-1,2(\mathrm{mg} / \mathrm{dL})$ \\
\hline BUN & 7 & 5 & 3 & 7-17 (mg/dL) \\
\hline Glucosa & 81 & 78 & 79 & 74-100 (mg(dL) \\
\hline CK & & - & 204 & 30-135 (U/L) \\
\hline CK-MB & - & - & 2 & $<16(\mathrm{U} / \mathrm{L})$ \\
\hline Troponina I & $\longrightarrow$ & - & 0,04 & $<0,08(\mathrm{ng} / \mathrm{ml})$ \\
\hline SGOT & 29 & 23 & 19 & $14-36(\mathrm{U} / \mathrm{L})$ \\
\hline SGPT & - & - & 14 & $9-52(\mathrm{U} / \mathrm{L})$ \\
\hline GGT & - & - & 7 & $12-43(\mathrm{U} / \mathrm{L})$ \\
\hline Fosfatas alcalina & 76 & 54 & 52 & 38-126 (U/L) \\
\hline LDH & 275 & 254 & 241 & 313-618 (U/L) \\
\hline Bilirrubina total & 0,2 & 0,2 & 0,2 & $0,2-1,3(\mathrm{mg} / \mathrm{dL})$ \\
\hline Leucocitos & 3570 & 8000 & 15000 & $4000-1000 \times \mathrm{mm}^{3}$ \\
\hline Hematocrito & 39 & 31,6 & 32 & $37-44(\%)$ \\
\hline Plaquetas & 212000 & 228000 & 260000 & $150000-400000 \times \mathrm{mm}^{3}$ \\
\hline Proteína $\mathrm{C}$ reactiva & - & 11,2 & 13,4 & $<10(\mathrm{mg} / \mathrm{L})$ \\
\hline Procalcitonina & - & - & 0,4 & $<0,5(\mathrm{ng} / \mathrm{ml})$ \\
\hline Acido valproico & - & - & 41 & $50-100(\mu \mathrm{g} / \mathrm{ml})$ \\
\hline
\end{tabular}


láctico 4,1 mmol/L. En ese momento, la paciente se encontraba estable en lo hemodinámico, con una presión arterial media (PAM) de $80 \mathrm{mmHg}$, frecuencia cardíaca de 70 lat/min y una diuresis de 60 a $80 \mathrm{ml} / \mathrm{h}$, sin evidencias de hipoperfusión clínica (piel tibia y con adecuado llene capilar).

Luego de la estabilización inicial, se realiza una TAC de cerebro de control, en la que se aprecian cambios post-quirúrgicos: lecho operatorio sin hematoma y línea media conservada. El electroencefalograma descartó la existencia de actividad epileptógena y evidenció un ritmo lento generalizado atribuible a la sedación.

Se mantuvieron las medidas habituales de manejo post-quirúrgico, garantizando una adecuada presión de perfusión cerebral. Durante las siguientes horas la paciente no presentó nuevas crisis convulsivas motoras.

A las 21:00 horas, un nuevo control gasométrico pone en evidencia el agravamiento de la acidosis láctica: $\mathrm{pH} 7,12 ; \mathrm{PaCO}_{2} 31 \mathrm{mmHg}$; bicarbonato $10 \mathrm{mmol} / \mathrm{L} ; \mathrm{BE}-18 \mathrm{mmol} / \mathrm{L}$ y ácido láctico $9,9 \mathrm{mmol} / \mathrm{L}$; pero con una saturación venosa central de $86 \%$. El electrocardiograma no mostraba cambios sugerentes de isquemia miocárdica y las enzimas cardiacas fueron normales.

En este contexto, se decide realizar monitorización hemodinámica invasiva con un catéter de arteria pulmonar, encontrándose una presión de oclusión en la arteria pulmonar (POAP) de 10 $\mathrm{mmHg}$, un índice cardíaco de $5,2 \mathrm{~L} / \mathrm{min} / \mathrm{m}^{2}$, una resistencia vascular sistémica indexada de 1174 dinas $\cdot \mathrm{seg} \cdot \mathrm{cm}^{5} \cdot \mathrm{m}^{2}$ y una saturación venosa mixta (Satvm) de 77\%. Se continuó el aporte de fluidos trazándose como meta una POAP de 14 a 16 mmHg y se asoció noradrenalina con el objeto de mantener una PAM de 90 a $100 \mathrm{mmHg}$. Adicionalmente, se incrementó la infusión de midazolam a $20 \mathrm{mg} / \mathrm{h}$ y se reinició la administración de propofol a $3,5 \mathrm{mg} / \mathrm{kg} / \mathrm{h}(58 \mu \mathrm{g} / \mathrm{kg} / \mathrm{min})$ para alcanzar un nivel de 2 en la escala de sedación-agitación descrita por Riker ${ }^{16}$.

Sin embargo, pese a mantenerse estable en lo macro y microhemodinámico y sin presentar disfunciones orgánicas, en el control de las 00:20 se constató llamativamente, un empeoramiento de la acidosis láctica: pH 7,0 y ácido láctico 8,5 con Satvm $80 \%$. No fue posible identificar una causa evidente que explicase la evolución clínica de la paciente, se mantuvo terapia de soporte. En las horas siguientes, no se observó un cambio significativo de la acidosis metabólica (Tabla 2).

Finalmente, se sospechó que la administración de propofol podría ser la causa desencadenante y la perpetuadora de la acidosis láctica, por lo que se decidió suspender la infusión a las 08:30,

Tabla 2. Evolución de la infusión de propofol y balance ácido base

\begin{tabular}{|c|c|c|c|c|c|c|}
\hline $\begin{array}{l}\text { Variables Fecha } \\
\text { Hora }\end{array}$ & $\begin{array}{r}13 / 06 / 06 \\
17: 00\end{array}$ & $\begin{array}{r}13 / 06 / 06 \\
21: 00\end{array}$ & $\begin{array}{r}14 / 06 / 06 \\
00: 20\end{array}$ & $\begin{array}{r}14 / 06 / 06 \\
06: 00\end{array}$ & $\begin{array}{r}14 / 06 / 06 \\
12: 18\end{array}$ & $\begin{array}{r}14 / 06 / 06 \\
19: 00\end{array}$ \\
\hline \multicolumn{7}{|l|}{ Propofol (mg/kg) } \\
\hline $\mathrm{pH}$ & 7,34 & 7,12 & 7,06 & 7,26 & 7,53 & 7,45 \\
\hline $\mathrm{PaCO}_{2}(\mathrm{mmHg})$ & 36 & 31 & 30 & 28 & 24 & 35 \\
\hline $\mathrm{HCO}_{3}(\mathrm{mmol} / \mathrm{L})$ & 19 & 10 & 8,5 & 12,5 & 19,1 & 21 \\
\hline $\mathrm{BE}(\mathrm{mmol} / \mathrm{L})$ & $-6,4$ & -18 & $-21,6$ & $-12,9$ & -3 & $-1,2$ \\
\hline Lactato $(\mathrm{mmol} / \mathrm{L})$ & 4,1 & 9,9 & 8,5 & 7,5 & 2,9 & 1,6 \\
\hline \multirow[t]{2}{*}{ Sat. Venosa (\%) } & - & 86 & 71 & 80 & 78 & 79 \\
\hline & & (central) & (mixta) & (mixta) & (mixta) & (mixta) \\
\hline $\mathrm{IC}\left(\mathrm{L} / \mathrm{min} / \mathrm{m}^{2}\right)$ & & $\longrightarrow$ & 3,7 & 4,0 & 4,2 & 4,4 \\
\hline \multirow[t]{2}{*}{ P. llenado (mmHg) } & & 10 & 14 & 15 & 14 & 12 \\
\hline & & (PVC) & (POAP) & (POAP) & (POAP) & (POAP) \\
\hline
\end{tabular}

IC: índice cardiaco, P. Llenado: presiones de llenado, PVC: presión venosa central, POAP: presión de oclusión en la arteria pulmonar. 
habiendo completado 8 horas adicionales después de la cirugía. La condición hemodinámica continuó estable durante la mañana. Cuatro horas más tarde se realizó un nuevo control gasométrico, en el que se pudo apreciar la corrección de la acidosis láctica: pH 7,53 y ácido láctico 2,9 mmol/ $\mathrm{L}$ con $\mathrm{PaCO}_{2} 24 \mathrm{mmHg}$. A las 19:00, once horas después de la suspensión del propofol, el acido láctico ya se había normalizado: $1,6 \mathrm{mmol} / \mathrm{L}$ (Tabla 2). Posteriormente, la paciente tuvo una evolución favorable, sin complicaciones asociadas a la descompensación metabólica. No volvió a presentar crisis convulsivas, pudiendo ser extubada y trasladada de la UCI en buenas condiciones.

\section{DisCUSIÓN}

Desde comienzo de los 90, múltiples reportes pediátricos han alertado sobre los riesgos que conlleva la administración de propofol en altas dosis y por tiempo prolongado. De hecho, varios autores intentaron establecer una asociación directa entre la infusión de propofol y algunas muertes ocurridas en este grupo de enfermos $4,5,17$. En la última década se han documentado varios casos de acidosis láctica asociada a la infusión de propofol en pacientes adultos ${ }^{10,18,19}$.

El SIP es una entidad rara pero a menudo fatal, donde la administración de propofol ocasiona un bloqueo del ingreso de ácidos grasos libres de cadena larga a la mitocondria. Adicionalmente, impide la adecuada oxidación intramitocondrial e interfiere con la cadena respiratoria, el resultado es la imposibilidad de la utilización de ácidos grasos de cadena media y corta. Esta grave alteración metabólica conduce a una baja producción energética y por tanto a un desbalance entre el aporte y la demanda de energía, generando acidosis láctica y en estados avanzados, necrosis del músculo cardíaco y periférico ${ }^{20}$.

Algunos de los factores de riesgo asociados con el SIP son: infección respiratoria alta, infusión por más de 48 h en dosis mayores a $5 \mathrm{mg} / \mathrm{kg} / \mathrm{h}$, empleo de corticoides o catecolaminas y bajo aporte de hidratos de carbono ${ }^{15,21}$. Varios autores han encontrado una mayor frecuencia de SIP en pacientes neurocríticos (especialmente en TEC grave) ${ }^{14,15}$, probablemente en relación a los altos niveles de catecolaminas endógenas liberadas por el sistema nervioso simpático y eventualmente por la administración de catecolaminas exógenas en algunos de estos enfermos. Adicionalmente, algunos investigadores apoyados en estudios experimentales, han planteado la hipótesis de que para el desarrollo del SIP se requiere la presencia de una condición predisponente, como patología del SNC, y un factor gatillante, como la administración o interacción de algunos compuestos como catecolaminas o esteroides con propofol ${ }^{15}$. Nuestra paciente reunía todas las condiciones para encontrarse en alto riesgo de desarrollar SIP, ya que había sido sometida a una intervención neuroquirúrgica mayor, como fue la resección de su MAV y además había recibido catecolaminas exógenas y esteroides durante la cirugía y en el período postoperatorio inmediato.

La paciente mantuvo una macrohemodinamia estable, con presiones de perfusión adecuadas e índice cardiaco y saturación venosa mixta dentro rangos normales (Tabla 2), sin evidencia clínica de hipoperfusión que pudiera explicar la acidosis láctica. Adicionalmente, no presentó alteraciones clínicas ni de laboratorio compatibles con un cuadro infeccioso activo, se mantuvo afebril, sin desviación a la izquierda en el hemograma, con proteína $\mathrm{C}$ reactiva y procalcitonina dentro de rangos normales, considerando el procedimiento quirúrgico (Tabla 1).

La acidosis láctica ha sido catalogada como un marcador precoz en el desarrollo de $\operatorname{SIP}^{22}$. En el presente caso, el retiro oportuno de la infusión de propofol revirtió rápidamente la acidosis láctica, lo que podría explicar porque nuestra paciente no desarrolló rabdomiolisis, bradicardia, ni depresión miocárdica.

La paciente no tenía antecedentes de insuficiencia renal crónica y durante el período perioperatorio no se evidenció un deterioro agudo de la función renal que pudiera explicar la acidosis metabólica. La función hepática siempre fue normal, lo que descartaría la acumulación de ácido láctico como causa de la acidosis metabólica (Tabla 1). Aunque se ha reportado el desarrollo de acidosis láctica en el contexto de una sobredosis aguda de ácido valproico $(430 \mathrm{mg} / \mathrm{kg})^{23}$, no se ha documentado tal complicación durante la administración de dosis terapéuticas. Nuestra paciente tuvo niveles plasmáticos de $41 \mathrm{mg} / \mathrm{ml}$ (rango normal: $50-100 \mathrm{mg} / \mathrm{ml}$ ) durante el episodio de acidosis láctica, siendo por tanto improbable que 
este fármaco fuera el inductor del cuadro. Concordante con ello y a pesar de mantenerse la administración de ácido valproico, la acidosis láctica se corrigió rápidamente luego de suspendida la infusión de propofol.

El presente caso es relevante por varias razones. En primer lugar, constituye la primera comunicación en nuestro medio de acidosis láctica severa asociada a una infusión de propofol de corta duración. En segundo lugar, resalta la importancia de tener presente en el trabajo diagnóstico diferencial, la presencia de esta complicación

\section{REFERENCIAS}

1. Withington D, Decell M, Al Ayed T. A case of propofol toxicity: further evidence for a causal mechanism. Paediatr Anaesth 2004; 14: 505-08.

2. Glen J, Hunter S. Phamacology of an emulsion formulation of ICI 35.868. Br J Anaesth 1984; 56: 617-26.

3. Bray R. Propofol infusion syndrome in children. Paediatr Anaesth 1998; 8: 491-99.

4. Cray S, Robinson B, Cox P. Lactic Acidemia and bradyarrhythmia in a child sedated with propofol. Crit Care Med 1998; 26: 2087-92.

5. Hanna J, Ramundo M. Rhabdomyolysis and hipoxia associated with prolonged propofol infusion in children. Neurology 1998; 50: 301-03.

6. НатсH D. Propofol-infusion syndrome in children. Lancet 1999; 353: 1117-18.

7. Kelly D. Propofol infusion syndrome. J Neurosurg 2001; 95: 925-26.

8. Wolf A, Weir P, Segar P, Stone J, Shield J. Impaired fatty acid oxidation in propofol infusion syndrome. Lancet 2001; 357: 606-7.

9. Cremer O, Moons K, Bouman E, Krujjswijk J, Smet A, KalKman C. Long-term propofol infusion and cardiac failure in adult head-injury patients. Lancet 2001; 357: 117-18.

10. Perrier N, Baerga-varela Y, Murray M. Death related to propofol use in an adult patient. Crit Care Med 2000; 28: 3071-74.

11. Badr A, Mychaskiw G, EichHorn J. Metabolic acidosis associated with a new formulation of propofol. Anesthesiology 2001; 94: 536-38.

12. Burow B, Johnson M, Packer D. Metabolic acidosis associated with propofol in the absence of other causative factors. Anesthesiology 2004; 101: 239-41. infrecuente pero potencialmente letal, sobre todo en pacientes que reúnan los factores de riesgo previamente comentados y que desarrollen acidosis láctica de causa no precisada. En último término, enfatiza el temprano reconocimiento de este síndrome, ya que ello puede permitir la oportuna corrección del mismo, antes que se establezcan otras alteraciones orgánicas y metabólicas. Esto adquiere trascendencia si consideramos que habitualmente la expresión completa del SIP suele traducir un estado avanzado de su evolución y muchas veces irreversible.

13. Eiksen J, Povey H. A case of suspected nonneurosurgical adult fatal propofol infusion syndrome. Acta Anaesthesiol Scand 2006; 50: 117-19.

14. Fudickar A, Bein B, Tonner P. Propofol infusion syndrome in anaesthesia and intensive care medicina. Curr Opin Anaesthesiol 2006; 19: 404-10.

15. Vasile B, Rasulo F, Candiani A, Latronico N. The pathophysiology of propofol infusion syndrome: a simple name for a complex syndrome. Intensive Care Med 2003; 29: 1417-25.

16. Riker RR, Picard JT, Fraser GL. Prospective evaluation of the Sedation-Agitation Scale for adult critically ill patients. Crit Care Med 1999; 27: 1325-29.

17. Parke T, Stevens J, Rice A, Greenaway C, Bray R, SMITH P ET AL. Metabolic acidosis and fatal myocardial failure after propofol infusion in children: Five cases reports. BMJ 1992; 305: 613-6.

18. Marinella M. Lactic acidosis associated with propofol (communication to the editor). Chest 1996; 109: 292.

19. Cannon M, Glazier S, Bauman L. Metabolic acidosis, rhabdomyolysis and cardiovascular collapse after prolonged propofol infusion. J Neurosurg 2001; 95: 1053-56.

20. Motsch J, Roggenbach J. Propofol infusion syndrome. Anaesthesist 2004; 53: 1009-22.

21. Salengros JC, Velghe-lenelle CE, Bollens R, Engelman E, Barvais L. Lactic acidosis during propofolremifentanil anesthesia in an adult. Anesthesiology 2004; 101: 241-43.

22. Koch M, De Backer D, Vincent JL. Lactic acidosis: an early marker of propofol infusion syndrome? (communication to the editor). Intensive Care Med 2004; 30: 522.

23. Blayac D, Roch A, Michelet P, De Francheschi E, AufrRAY J. Deep lactic acidosis after valproato selfpoisoning. Ann Fr Anesth Reanim 2004; 23: 1007-10. 